\title{
Mise au point d'un dosage sélectif des différents types de mucines gastro-intestinales: utilisation de réactifs histochimiques
}

\author{
N Fontaine, JC Meslin
}

INRA, laboratoire de nutrition et sécurité alimentaire, 78352 Jouy-en-Josas Cedex, France

(Reçu le 15 septembre 1993 ; accepté le 14 mars 1994)

\begin{abstract}
Résumé - Une méthode, transposée des tests histochimiques utilisés pour les mucines, est proposée pour évaluer par spectrocolorimétrie les mucines neutre acide et sulfatée. Les colorations respectives sont l'acide périodique-Schiff (APS), le bleu Alcian (BA) pH 2,5 et le bleu Alcian (BA) pH 0,5. Les échantillons de mucines sont extraits de grattages de muqueuses et de contenus intestinaux de rats axéniques ou de mucus gastrique de porc d'origine commerciale. Une fraction enrichie en mucine est préparée et lyophilisée et son contenu en protéines est déterminé. Chaque type de mucine est analysé par spectrophotométrie après coloration et précipitation au mélange de Carnoy modifié. Les colorations d'histochimie appliquées, contrôlées en électrophorèse, après traitement par le mélange de Carnoy modifié, restent spécifiques des types de mucines contenus dans ces mucines et ne révèlent pas les protéines contaminantes.
\end{abstract}

mucines / intestin / évaluation relative

Summary - Spectrocolorimetric assay for the 3 types of gastro-intestinal mucins using histochemical stains. A method, transposed from the mucin histochemical stainings, was proposed to evaluate neutral, acidic and sulphated mucins by spectrocolorimetry. Stainings used were periodic acid/Schiff (PAS), Alcian blue (AB) pH 2.5, and Alcian blue (AB) pH 0.5. Mucin samples were extracted from mucosal scrapings, from intestinal contents of germ-free rats or from commercial pig gastric mucin. A mucin-enriched fraction was obtained and lyophilised and the protein content was determined. Each mucin type was spectrophotometrically analysed after staining and precipitation by slightly modified Carnoy fixative. The histochemical stainings used here, after modification by Carnoy fixative treatment, were, as shown by electrophoretic controls, specific for mucin types contained in these mucins without protein contaminant interference.

mucin / intestine / quantitative evaluation 


\section{INTRODUCTION}

Le mucus intestinal a un rôle particulièrement important pour la lubrification et la protection de l'épithélium (Neutra et Forstner, 1987). La mucine est composée de $80 \%$ de chaînes glycaniques très diversifiées en longueur et en structure oligosaccharidiques (Roussel et al, 1988). Sa composition en polypeptides est aussi variable et est de l'ordre de $20 \%$.

Des modifications quantitatives et/ou qualitatives, en particulier les changements qui apparaissent dans les 3 classes de mucines (neutre, acide, acide sulfatée) au cours de l'évolution de certaines maladies (Bogomoletz et al, 1984 ; Bogomoletz et al, 1987 ; Rhodes, 1989), ou de changements alimentaires (Satchithanandam et al, 1990 ; Turck et al, 1993) sont de grande importance pour la suite du déroulement des fonctions digestives. Une attention particulière est portée à l'altération des taux relatifs des mucines sulfatées ou sulfomucines, et des mucines acides ou sialomucines (Filipe et Branfoot, 1974 ; Filipe, 1979 ; Filipe et Fenger, 1979).

Nos observations en histologie (Meslin et al, 1993) montrent que le nombre de cellules à mucus et la nature du mucus qu'elles renferment peuvent varier selon la nature de l'aliment et l'état bactérien des animaux.

Divers travaux rapportent des modifications du nombre des cellules à mucus à différents sites intestinaux dans la muqueuse en relation avec l'âge des animaux ou la nature des aliments ingérés, plus particulièrement selon le type de fibres présentes dans cet aliment. Ces observations histologiques quantitatives sont parfois accompagnées d'observations semi-quantitatives sur la colorabilité des contenus intestinaux.

Les laboratoires qui disposent d'anticorps anti-mucus produits sur place effectuent une immuno-titration du mucus sécrété, présent dans le milieu de culture (Roumagnac et Laboisse, 1989) ou bien, lorsque le laboratoire l'a fabriqué, un anticorps dirigé contre un certain type de mucus (Bara et al, 1990; Yamori et al, 1989).

Perumal et al (1966) ont utilisé du ${ }^{35} \mathrm{~S}$ dans l'étude de la biosynthèse des mucopolysaccharides en relation avec les vitamines. Mantle et al (1989) utilisent des radioéléments ${ }^{3} \mathrm{H}$ et ${ }^{14} \mathrm{C}$ pour étudier la synthèse et la dégradation du mucus in vivo et Maoret et al (1989) pour caractériser le mucus in vitro. Ces méthodes présentent divers inconvénients ; en particulier, elles ne permettent pas d'estimer séparément les 3 classes de mucines sur un même échantillon.

Des méthodes utilisant des lectines, qui sont particulièrement spécifiques de certains sucres, sont utilisées (Doehrn et al, 1992 ; Ellinger et Pavelka, 1992 ; Suzaki et Kataoka, 1992 ; Szentkuti et al, 1992 ; Ishikawa et al, 1993). Ces lectines sont particulièrement onéreuses et aucune ne permet dans la mucine de quantifier globalement les fractions de mucine neutre, acide et sulfatée.

Les méthodes histologiques utilisent le bleu Alcian (BA) pour visualiser les mucines acides (sialomucines et sulfomucines) à $\mathrm{pH}$ 2,5 mais ce colorant n'est pas toujours spécifique dans les conditions biochimiques. Le seul test colorimétrique relatif aux glycoprotéines neutres contenues dans le mucus est celui de Mantle et Allen (1978) qui utilise la technique à l'acide périodiqueschiff (APS). Par contre Roe et al (1989) ont mesuré l'intensité de coloration de coupes histologiques après solubilisation de ces dernières en utilisant une méthode modifiée combinant l'APS et le bleu Alcian après saponification. Cela leur permet de mesurer en intensité relative la colorabilité du mucus pour un type bien défini de mucines sur les critères histochimiques (mucines neutre, acide, sulfatée) mais il n'existe pas de mesures biochimiques transposées des méthodes histologiques pour 
ces 3 classes de mucines dans diverses conditions physiologiques.

Le but de cette étude est de quantifier les 3 classes de mucines présentes dans le mucus par transposition des techniques histochimiques appliquées dans ce domaine. L'objectif ultérieur est d'appliquer cette méthode à diverses conditions physiologiques et/ou nutritionnelles sur des échantillons de muqueuses et de contenus intestinaux. Pour cela, on a utilisé de la mucine prélevée à partir de l'intestin de rat axénique, provenant à la fois des muqueuses et des contenus intestinaux et du mucus gastrique de porc du commerce. Le rat axénique a été choisi du fait de l'abondance du mucus dans ses contenus intestinaux, non dégradé du fait de l'absence de bactéries (Hoskins et Zamcheck, 1968). Ceci permet les conditions les plus favorables pour la mise au point de la technique avec une meilleure sensibilité du dosage.

\section{MATÉRIEL ET MÉTHODES}

\section{Source de mucus}

\section{Mucus intestinal de rat axénique}

Cinq rats axéniques mâles de souche Fischer F344 du centre d'élevage de l'INRA UEPSD, âgés de 3 mois, de poids moyen $350 \pm 22 \mathrm{~g}$, élevés dans un environnement de température et d'humidité relative de l'animalerie contrôlées (21 $\pm 2^{\circ} \mathrm{C}, 60 \pm 5 \%$, respectivement) et sous un cycle lumineux aussi contrôlé ( $12 \mathrm{~h}$ lumière/obscurité) sont utilisés pour cette expérience.

Les rats sont placés sur cages grillagées et sont maintenus en isolateurs vinyle. lis ont reçu l'aliment standard commercial UAR ad libitum. Cet aliment conditionné en sacs plastiques scellés sous vide a été stérilisé par irradiation gamma à 40 kGy.

Les rats reçoivent sous anesthésie légère à l'éther une injection intrapéritonéale de $0,2 \mathrm{ml}$ de pilocarpine (solution à $1 \%$ dans l'eau distillée) (Sigma, France), pour provoquer la sécrétion de mucus dans la lumière intestinale. Après un temps d'action de $10 \mathrm{~min}$, les animaux sont sacrifiés par anesthésie profonde à l'éther.

Les contenus intestinaux de l'intestin grêle, du caecum et du côlon sont recueillis par lavage de la lumière intestinale par du $\mathrm{NaCl}$ à $0,9 \%, \mathbf{p H}$ 7 , EDTA $0,1 \mathrm{mM}$, additionné d'inhibiteurs des protéases (Azide de $\mathrm{Na} \mathrm{0,02 \%} \mathrm{PMSF} \mathrm{0,1} \mathrm{mM).}$ Les muqueuses des mêmes sites intestinaux sont recueillies par grattage et réunies avec les contenus. L'ensemble contenus et grattages des muqueuses est complété à un volume de $200 \mathrm{ml}$ par le $\mathrm{NaCl} 0,9 \%$ pour les 5 rats, tous segments réunis.

\section{Mucus gastrique de porc, fraction II Sigma, lot 2372}

Le mucus est utilisé tel quel, sans purification ultérieure. Les solutions sont généralement effectuées à $1 \mathrm{mg}$ par $\mathrm{ml}$ dans l'eau distillée ou le PBS $\mathrm{NaCl} 0,9 \% \mathrm{pH} 7,4$. Cela correspond à $87,2 \mu \mathrm{g}$ de protéines par $\mathrm{ml}$.

\section{Préparation de la fraction enrichie en mucine}

Pour l'extraction et la purification de la mucine, on a utilisé la technique de Miller et Hoskins (1981), légèrement modifiée : une seule précipitation alcoolique au lieu de précipitations multiples.

Les prélèvements sont homogénéisés à l'ultraturrax quelques secondes puis centrifugés à $700 \times g$ et le surnageant est recentrifugé à 27 $000 \mathrm{xg}$. Cela permet d'éliminer les particules alimentaires insolubles, les cellules desquamées, et de récupérer la fraction soluble contenant la mucine.

Pour effectuer la précipitation alcoolique de la mucine, le surnageant est mis en contact avec de l'alcool éthylique pour obtenir une concentration finale de $60 \%(\mathrm{v} / \mathrm{v})$ durant 1 heure à $-20^{\circ} \mathrm{C}$, puis centrifugé à $47800 \mathrm{xg} 20 \mathrm{~min}$ à $-20^{\circ} \mathrm{C}$.

Le surnageant est éliminé et le précipité est resolubilisé dans le tampon $\mathrm{NaCl} 0,9 \%$ plus inhibiteurs. Il est dialysé ( 5 fois $2 h$ ) dans l'eau distillée à $4^{\circ} \mathrm{C}$. Ceci élimine les composés dialysables qui auraient pu être entraînés avec la mucine. La fraction non dialysable est alors lyophilisée et stockée à $-20^{\circ} \mathrm{C}$. Le poids du lyophilisat ainsi recueilli est de $420 \mathrm{mg}$. 


\section{Dosage des trois classes de mucines}

Au moment du dosage, le produit lyophilisé est solubilisé à une concentration de $1 \mathrm{mg} / \mathrm{ml}$ par dilution dans l'eau distillée. Cela correspond à $70 \mu \mathrm{g}$ de protéines par $\mathrm{ml}$ chez le rat. Des gammes de concentrations croissantes sont effectuées, colorées puis la mucine est précipitée par un mélange de Carnoy modifié (éthanol absolu $6 \mathrm{v}$, formaldéhyde à $40 \% 3 \mathrm{v}$, acide acétique RP 1 v) et le précipité coloré est redissous (comme il est dit plus loin). Le dosage est évalué au spectrophotomètre (Shimatsu UV 240 Roucaire) à 570 $\mathrm{nm}$ pour l'acide périodique-Schiff et à $600 \mathrm{~nm}$ pour le bleu Alcian.

\section{Mucine neutre (APS+)}

On applique la méthode de Mantle et Allen (1978).

La gamme va de 0 à $0,1 \mathrm{mg} / \mathrm{ml}$. La lecture est effectuée au spectrophotomètre à $570 \mathrm{~nm}$.

\section{Mucine acide (BA pH 2,5+)}

À ce pH le bleu Alcian met en évidence la mucine acide composée à la fois de sialomucine et de sulfomucine (Ganter et Jollès, 1969-1970).

La gamme est effectuée de 0 à $0,5 \mathrm{mg} / \mathrm{ml}$ qsq $1 \mathrm{ml}$ eau, on ajoute $60 \mu \mathrm{l}$ d'acide acétique et 400 $\mu \mathrm{l}$ de bleu Alcian $8 \mathrm{GX}(\mathrm{Cl} 74240)$ [Sigma] à $\mathrm{pH}$ $2,5(1 \%$ dans l'acide acétique à $3 \%)$. Après un temps de contact de $15 \mathrm{~min}$, on ajoute $500 \mu \mathrm{lde}$ mélange de Carnoy modifié puis on centrifuge à $9500 \mathrm{xg}$ pendant $10 \mathrm{~min}$; le surnageant est jeté, on rajoute au précipité coloré $1,7 \mathrm{ml}$ d'eau distillée, $60 \mu$ de Triton $\times 100$ à $20 \%$ et $240 \mu$ l'acide acétique à $20 \%$ pour avoir un $\mathrm{pH}$ de 2,5 . Le volume final est de $2 \mathrm{ml}$. L'ensemble est traité aux ultrasons (Cuve Bransonic) pendant $1 \mathrm{~min}$, puis la lecture est effectuée à $600 \mathrm{~nm}$.

\section{Mucine sulfatée (BA pH 0,5+)}

Pour cette valeur de $\mathrm{pH}$, le bleu Alcian ne révèle que la sulfomucine ou mucine strictement sulfatée (Ganter et Jollès, 1969-1970).

La gamme est effectuée de 0 à $0,5 \mathrm{mg} / \mathrm{ml}$, qsq $1 \mathrm{ml}$ par de l'eau distillée ; on ajoute $0,1 \mathrm{ml}$ d'HCl $5,5 \mathrm{~N}, 400 \mu$ de bleu Alcian à pH $0,5(0,5 \%$ dans $\mathrm{HCl} 0,5 \mathrm{~N}$ ); on laisse en attente $15 \mathrm{~min}$, on ajoute $500 \mu \mathrm{l}$ de mélange de Carnoy modifié puis on centrifuge à $9500 \times$ g pendant $10 \mathrm{~min}$; le surnageant est jeté et l'on rajoute au précipité coloré $1,5 \mathrm{ml}$ d'eau distillée $+60 \mu \mathrm{l}$ de Triton $\mathrm{X} 100$ à $20 \%$ et $440 \mu \mathrm{l}$ d' $\mathrm{HCl} 2 \mathrm{~N}$ pour avoir un $\mathrm{pH}$ de 0,5 . Le volume final est de $2 \mathrm{ml}$. L'ensemble est traité aux ultrasons pendant $1 \mathrm{~min}$ et la lecture est effectuée à $600 \mathrm{~nm}$.

\section{Dosage des protéines}

Le contenu en protéines est évalué par la méthode de Lowry modifiée (Bensadoun et Weinstein, 1976). Le mucus gastrique de porc renferme 87,2 $\mu \mathrm{g}$ de protéines par $\mathrm{mg}$, et la mucine d'intestin de rat axénique, après décharge par la pilocarpine, 70 $\mathrm{mg}$ de protéines par $\mathrm{mg}$ de mucine.

\section{Évaluation de la validité de la méthode par électrophorèse en gel de polyacrylamide avec SDS (SDS-PAGE)}

Plusieurs essais ont été effectués soit en gel de polyacrylamide à $7,5 \%$ surmonté d'un gel à $3 \%$ selon Laemmli (1970) (non montrés), soit en gradient de 3 à $8 \%$ selon Lambin et al (1976) en présence de sodium dodécyl sulfate (SDS).

Les échantillons de mucines $(50 \mu \mathrm{g}$ de protéine par puits) et les étalons protéiques (albumine [PM 67000], catalase [PM 232000], thyroglobuline [PM 669000] (Pharmacia), caséine (PM56000) [Sigma]) sont analysés par migration dans un gel de $0,1 \mathrm{~cm}$ d'épaisseur sur une plaque de $16 \mathrm{~cm} x$ $18 \mathrm{~cm}$. Le tampon utilisé est du Tris-glycine à $0,1 \%$ de SDS, pH 8,3 (Tris $0,05 \mathrm{M}$, glycine $0,384 \mathrm{M}$, réservoir du bas) et Tris-glycine $\mathrm{pH} 8,9$ (Tris 0,043 $M$, glycine $0,064 M$, réservoir du haut).

Le gel de séparation consiste en un gradient linéaire de polyacrylamide ( 3 à $8 \% \mathrm{p} / \mathrm{N}$ avec $30 / 0,8$ acrylamide bisacrylamide). Avant I'application sur le gel, les échantillons non chauffés, précipités ou non par le mélange de Carnoy modifié, sont dilués $(\mathrm{v} / \mathrm{v})$ avec une solution contenant du tampon Tris- $\mathrm{HCl}$ pH 6,8 2,5 glycérol 2, eau 0,5, SDS $10 \% 4, \beta$-mercaptoéthanol $1(\mathrm{v} / \mathrm{v} / \mathrm{v} / \mathrm{v} / \mathrm{v})$, bleu de bromophénol $0,01 \mathrm{p} / \mathrm{v}$.

Les électrophorèses sont conduites avec un courant de $4 \mathrm{~mA}$ puits, à voltage constant et à température ambiante, l'anode est au sommet du gel.

Après l'électrophorèse, les gels sont fixés au TCA à 12\% (Zacharius et al, 1969) et colorés comme suit. 


\section{Coloration des protéines}

Les protéines sont colorées par le bleu de Coomassie (Brillant blue R250) à $0,25 \%$ dans un mélange d'acide acétique-méthanol $50 \%$, eau (46/227/227 v/v/v) pendant $1 \mathrm{~h}$.

La partie glycosylée de l'échantillon est colorée par le BA et l'APS.

\section{Coloration des groupements acides}

Pour éliminer le SDS qui gêne la coloration et entraîne un fort bruit de fond, nous appliquons la méthode de Fairbanks et al (1971) en passant le gel dans des bains successifs d'un mélange d'acide acétique et de propanol puis dans l'acide acétique à $3 \%$ avant de faire réagir le $\mathrm{BA}$ pH 2,5 pendant $3 \mathrm{~min}$. Le gel est décoloré par l'acide acétique à $3 \%$ puis $7,5 \%$ ensuite par un léger passage dans l'eau de Javel diluée et l'eau du robinet (Heremans et al, 1959).

\section{Coloration des groupements neutres (APS+)}

Le gel débarrassé de l'excès de SDS comme précédemment (Fairbanks et al, 1971) est plongé dans un bain d'acide périodique à $0,5 \%$ pendant $50 \mathrm{~min}$ à $4^{\circ} \mathrm{C}$. Avant coloration par le réactif de Schiff, nous procédons comme Fairbanks en passant le gel dans des bains d'arsénite de $\mathrm{Na}$ pour éliminer le surplus d'acide périodique, ce qui évite le bruit de fond.

\section{ANALYSE STATISTIQUE}

La comparaison des droites de régression relatives aux dosages des mucines neutre, acide et acide sulfatée a été effectuée par la méthode de comparaison des coefficients de régression (Snedecor et Cochran, 1967).

\section{RÉSULTATS}

\section{Comparaison des électrophorèses}

Le contrôle de pureté de nos préparations en électrophorèse montre que le traitement de la mucine par le mélange de Carnoy modifié élimine un certain nombre de contaminants.

Alors que l'albumine, la catalase, la thyroglobuline (l'hémoglobine, l'aldolase, la ferritine [résultats non montrés sur cette électrophorèse]) sont toutes révélées par les colorants Coomassie et BA sans traitement, elles disparaissent toutes après traitement par le mélange de Carnoy modifié.

La préparation de mucine soluble de porc, sans traitement préalable par le mélange de Camoy, présente 4 bandes de contaminants. Trois bandes disparaissent après traitement par le mélange de Carnoy ; la préparation de mucine soluble de rat montre 6 bandes dont 3 disparaissent après traitement par le mélange de Carnoy (fig 1).

Les mucines étant de fort poids moléculaire, la molécule reste dans le gel à $3 \%$; la mucine n'est pas visible par la coloration au bleu de Coomassie. Cette coloration met en évidence en particulier les bandes de contaminants de petits poids moléculaires (fig 1) mais l'analyse des gels colorés au bleu Alcian montre que ces contaminants ne sont nullement révélés (fig 2). Seule la glycoprotéine apparaît colorée en bleu dans le gel à $3 \%$. De même avec l'APS, seul le haut du gel est coloré, montrant que seule la partie glucidique de la molécule a réagi (fig 3 ).

\section{Comparaison des pentes des gammes colorées exprimées en DO}

Cette méthode nous permet de comparer les pentes des droites obtenues sur des gammes dans chacune des classes de mucines étudiées (neutre, acide, acide sulfatée). II est ensuite possible pour chaque classe de mucine de comparer les $\mathrm{DO} / \mathrm{mg}$ des mucines provenant du mucus intestinal de rat axénique et du mucus gastrique de porc.

La figure 4 représente le dosage exprimé en $\mathrm{DO} / \mathrm{mg} / \mathrm{ml}$ de la mucine neutre conte- 

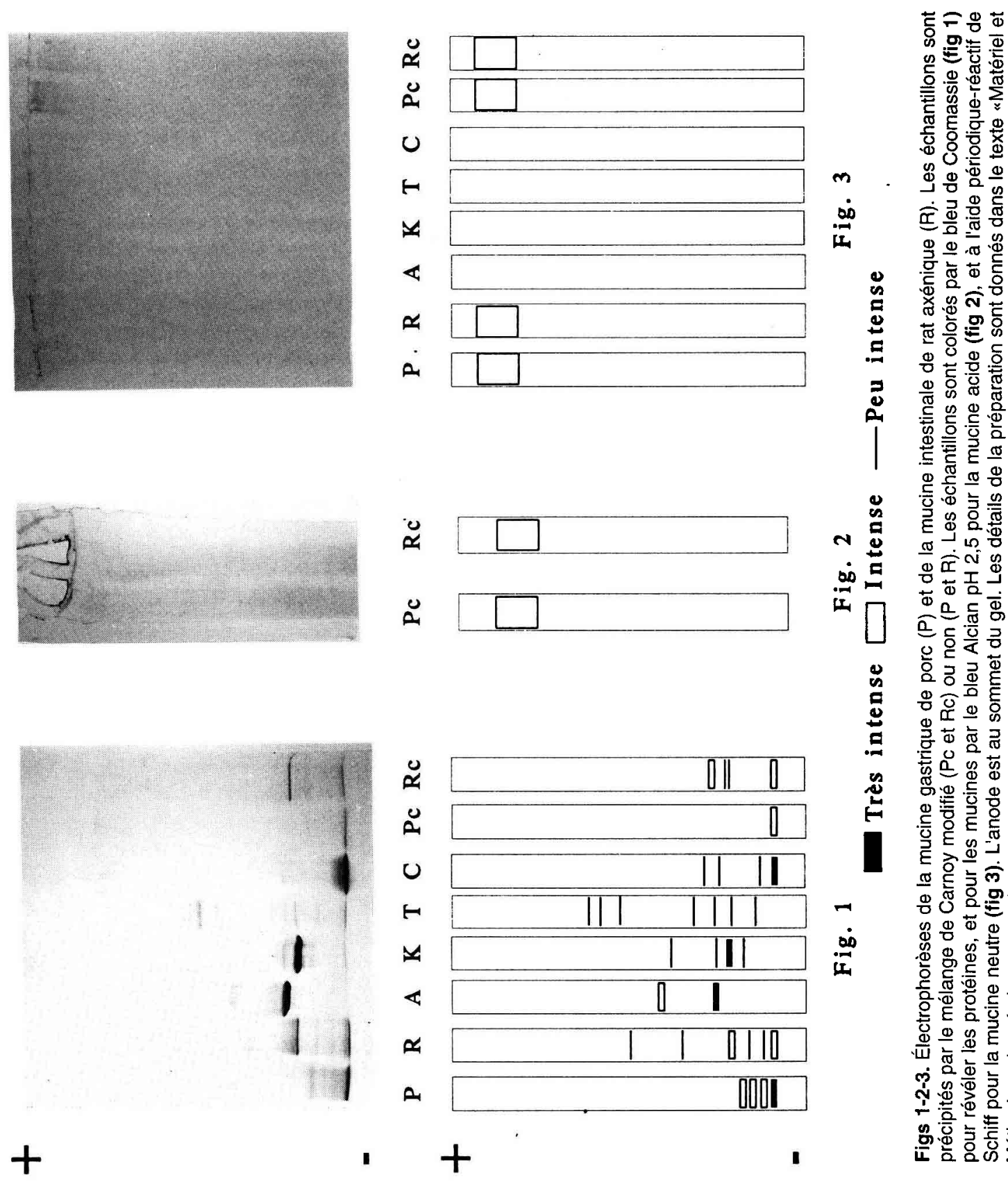

은

บ응

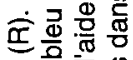

פ

흔 호응

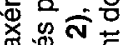

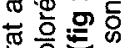

낭

응 등 응

它

든 등 는

吉至

동

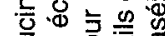

ह

(1)

ब立 $\cong$

도은

$\Phi$ 도응

믄 원

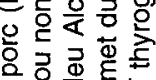

क О

号里

可市离焉

क क

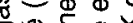

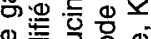

凹害号

距

즈ㅇㅡㅡ을

๘ 돋ㅎํ휘 음

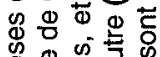

ब

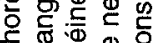

응 西

은

으

Ш丷木 西

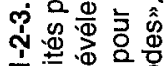

드원은

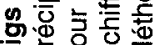

는응잉 
nue respectivement dans le mucus intestinal de rat axénique et dans le mucus gastrique de porc; le dosage des mucines acide et acide sulfatée est représenté sur la figure 5. Pour chacun de ces dosages, chaque point représente la valeur moyenne de 5 mesures et l'écart entre ces mesures est de $10 \%$.

Les estimations des valeurs de DO des mucines pour $1 \mathrm{mg}$ de la poudre lyophilisée sont regroupées dans le tableau l et sont calculées à partir des équations des droites de régression des figures 4 et 5 .

La quantité totale dosée pour les 5 rats représente $420 \mathrm{mg}$ de poudre lyophilisée, ce qui correspond à $29,4 \mathrm{mg}$ de protéines totales pour tout l'intestin. Pour le porc, afin de permettre la comparaison avec les résul-

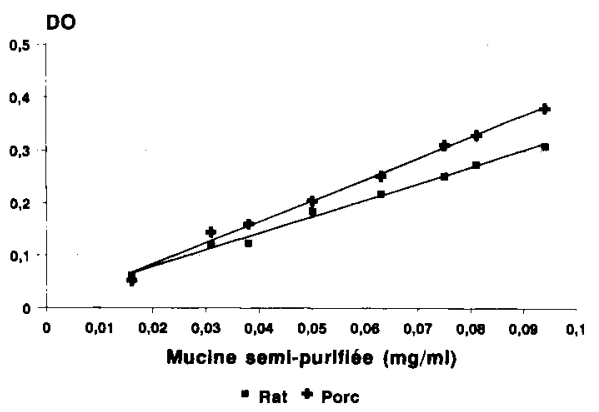

Fig 4. Dosage $(\mathrm{DO} / \mathrm{mg} / \mathrm{ml})$ de la mucine neutre contenue dans la mucine intestinale de rat axénique et dans la mucine gastrique de porc. tats obtenus chez le rat, le calcul est effectué en considérant une quantité de poudre lyophilisée équivalente à celle du rat, soit $420 \mathrm{mg}$, qui renfermerait alors $36,6 \mathrm{mg}$ de protéines.

Les résultats présentés dans le tableau I permettent de calculer que chez le rat la mucine neutre représente $37 \%$, la mucine acide $63 \%$, dont $47 \%$ de sialomucine et $16 \%$ de sulfomucine; chez le porc la mucine neutre représente $46 \%$, la mucine acide $54 \%$, se répartissant en $27 \%$ de sialomucine et $27 \%$ de sulfomucine. Compte tenu des variations estimées d'après les droites des dosages, nos résultats montrent que dans le mucus intestinal de rat et le mucus gastrique de porc les proportions de mucines ne sont pas différentes pour les

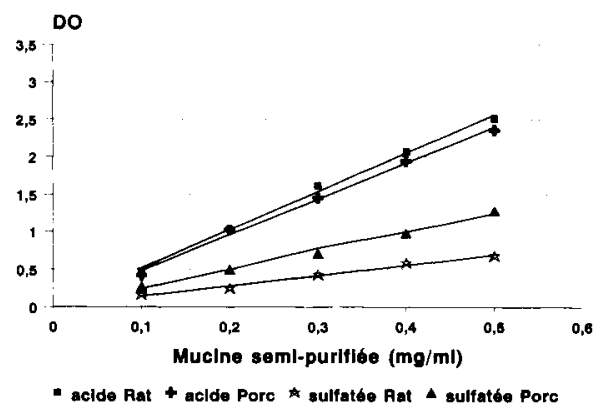

Fig 5. Dosage $(\mathrm{DO} / \mathrm{mg} / \mathrm{ml})$ des mucines acides et acides sulfatées contenues dans la mucine intestinale de rat axénique et dans la mucine gastrique de porc.

Tableau I. Bilan des mucines neutre, sialomucines et sulfomucines contenues dans le mucus intestinal de rat axénique et le mucus gastrique de porc (évaluation relative).

\begin{tabular}{lcccc}
\hline Mucines & \multicolumn{2}{c}{ Rat } & \multicolumn{2}{c}{ Porc } \\
& DO/mg & DO totale* & DO/mg & DO totale \\
\hline Neutre & & & & \\
Sialomucine & $3,26 \pm 0,20$ & 1370 & $4,06 \pm 0,22$ & 1705 \\
Sulfomucine & $4,43 \pm 0,39$ & 1747 & $2,75 \pm 0,33$ a & 1021 \\
& $1,85 \pm 0,33$ & 584 & $2,36 \pm 0,27$ a & 991 \\
\hline
\end{tabular}

* DO totale $=(\mathrm{DO} / \mathrm{mg}) \times 420 \mathrm{mg} .{ }^{\text {a }}$ Significativement différent $(P<0,05$ de la valeur correspondante du rat $)$. 
neutre et acide et diffèrent pour les mucines sulfatées, ces dernières étant plus importantes dans le mucus gastrique de porc que dans le mucus intestinal de rat.

\section{DISCUSSION}

L'analyse comparative des mucines intestinales de rat axénique effectuée dans cette étude porte sur un pool (intestin grêle, caecum et côlon, contenus et grattages des muqueuses réunis) provenant de 5 rats traités à la pilocarpine, afin de faire décharger le mucus dans la lumière du tractus digestif. Cela conduit à l'obtention d'une poudre lyophilisée d'un poids de $420 \mathrm{mg}$ contenant $29,4 \mathrm{mg}$ de protéines.

Les gammes de dosage des mucines permettent de calculer la quantité des mucines neutre (APS+), acide (sialomucine et sulfomucine $\mathrm{BA} \mathrm{pH} \mathrm{2,5+)} \mathrm{et} \mathrm{acide} \mathrm{stric-}$ tement sulfatée (sulfomucine $\mathrm{BA} \mathrm{pH} 0,5+$ ) contenues dans $1 \mathrm{mg}$ du lyophilisat initial, et exprimées en $\mathrm{DO} / \mathrm{mg}$. La sialomucine figurant dans le tableau I est obtenue par différence entre les valeurs de mucine acide et de mucine acide strictement sulfatée. II est ensuite possible de calculer les quantités correspondantes au pool initial de poudre lyophilisée recueillie. Celles-ci sont exprimées en DO totales.

Pour le porc, afin de permettre la comparaison avec les résultats obtenus chez le rat, le calcul est effectué en considérant une quantité de poudre équivalente à celle du rat, soit $420 \mathrm{mg}$, qui renfermerait alors 36,6 mg de protéines.

Des perturbations de la physiologie intestinale (diarrhées) ou des causes d'ordre pathologique sont susceptibles de modifier soit la sécrétion du mucus soit la nature et (ou) la quantité de mucines présentes dans le mucus (Filipe, 1979; Mian et al, 1979).

Le mucus colique humain est fortement sulfaté (Filipe, 1979) et il est connu que les hydrates de carbone sulfatés tendent à être résistants à la dégradation par les glycosidases (Mian et al, 1979). Ces aspects illustrent tout l'intérêt de pouvoir quantifier les classes de mucines présentes dans les mucines intestinales et, dans des expériences ultérieures, de pouvoir prendre en compte la part de dégradation de certaines de ces classes du fait de l'activité bactérienne (Miller et Hoskins, 1981).

Depuis les travaux de Allen (1978), Creeth (1978), Carlstedt et Sheehan (1984), Garner et al (1984), Neutra et Forstner (1987), la structure, la synthèse, les propriétés physiques et biologiques, le mécanisme de sécrétion des mucines intestinales ont pu être mieux connus ; mais à cause de nombreuses difficultés techniques associées à la récolte de ces mucines, qui se trouvent hautement contaminées par d'autres sécrétions et par les bactéries présentes dans la lumière intestinale, il reste encore beaucoup d'incertitudes à lever. Les revues de Laboisse et Bogomoletz (1989), Pasquier et Vatier (1990), Porchet et al (1991), Biol et al (1992), Hilkens et al (1992) font le point sur la question.

Dans l'étude rapportée dans la présente publication, nous développons une méthode pour l'estimation de la quantité des 3 classes de mucines (neutre, acide et sulfatée). Cette méthode n'a pas nécessité de purification poussée des mucines ; cette purification a été cependant évaluée par électrophorèse : l'échantillon de mucine reste bloqué dans le gel de faible concentration à l'origine du gel de l'électrophorèse. Au contraire, les protéines contaminantes sont détectées par des bandes de plus faible poids moléculaire révélées par le bleu de Coomassie (Allen et al, 1982). Dans les conditions de notre expérience, les bandes contaminantes ne sont pas révélées par le bleu Alcian et l'APS sur les électrophorèses et donc n'interfèrent pas lors du dosage spectrocolorimétrique des mucines neutre, acide et sulfatée, ce qui permet donc la 
caractérisation relative des 3 classes de mucines dans nos préparations. Les étalons protéiques ne sont montrés que pour prouver que les colorations au BA et à l'APS n'interfèrent pas avec ceux-ci. Notre but n'était nullement de faire le rapport des mucines avec les poids moléculaires des échantillons protéiques, et nos échantillons étant traités au $\beta$-mercaptoéthanol et SDS, les isoformes apparaissent dans nos étalons. Le traitement par le mélange de Carnoy modifié nous a permis une spécificité de la coloration au BA et à l'APS.

Dans les conditions normales, ces 3 classes de mucines sont sécrétées. La mucine neutre est détectée par la technique à l'acide périodique-Schiff (APS), tandis que les mucines acides (sialomucines et sulfomucines) peuvent être visualisées histochimiquement par le bleu Alcian à pH 2,5 et les mucines acides sulfatées (ou sulfomucines) par la méthode très spécifique à la diamine enrichie en fer (HID). Cette dernière méthode a été testée, mais elle nous a fourni des résultats très irréguliers, sans doute dus à l'instabilité des réactifs, ce qui ne nous a pas permis de poursuivre l'étude biochimique à l'aide de cette coloration. Nous avons donc remplacé cette coloration des mucines sulfatées par la coloration au bleu Alcian à pH 0,5 (Ganter et Jollès, 1969-1970).

La molécule glycoprotéique de mucus gastrique de porc est très complexe avec de nombreuses chaînes d'oses (Carlstedt et al, 1984, 1985). Cette molécule peut être divisée en 4 sous-unités par rupture des ponts disulfures et sa composition n'est pas la même que celle du mucus intestinal de rat (Allen, 1978). Cela explique en partie la distribution différente des 3 classes de mucines détectées ici par notre méthode de coloration et de quantification relative qui s'avère efficace dans les 2 cas, pour le rat et pour le porc.

La présente étude indique que notre échantillon total de mucine intestinale de rat axénique et un échantillon équivalent de mucus gastrique de porc renferment les mêmes proportions de mucines neutre et acide ; en revanche, il y a plus de mucine sulfatée dans le mucus gastrique de porc que dans la mucine intestinale de rat axénique.

Les travaux d'Allen (1978), Allen et al (1984), Hill (1986) fournissent des détails sur la composition de mucus gastrique de porc et ceux de Forstner et al (1973) sur celle des mucines intestinales de rat. Si l'on compare nos résultats avec ceux de ces auteurs, nous trouvons bien une proportion de protéines plus élevée ainsi qu'une plus grande quantité de mucine sulfatée dans le mucus gastrique de porc que dans celui de rat, ce qui va dans le même sens que les proportions de sulfate indiqués par ceux-ci.

Les résultats de cette étude montrent que l'on peut estimer en DO les mucines neutre, acide et sulfatée dans un extrait lyophilisé provenant d'une fraction enrichie en mucines. Nous envisageons d'appliquer cette méthode à des lots de rats dans diverses conditions microbiennes et nutritionnelles, d'une part sur les muqueuses, d'autre part sur les contenus intestinaux. Cela devrait permettre de faire le bilan du mucus stocké dans la paroi et de celui qui est présent dans les contenus intestinaux, c'est-à-dire sécrété.

Il est également envisagé de purifier les mucines par chromatographie afin d'obtenir un étalon de référence permettant la quantification des mucines en poids.

\section{REMERCIEMENTS}

Nous remercions l'UEPSD et Mme R Durao pour la fourniture et la maintenance des rats axéniques.

\section{RÉFÉRENCES}

Allen A (1978) Structure of gastrointestinal mucous glycoproteins and the viscous and gel-forming properties of mucus. Br Med Bull. $34,28-33$ 
Allen A, Bell A, Mantle M, Pearson JP (1982) The structure and physiology of gastrointestinal mucus. In: Mucus in Health and Diseases, part II, Advances in Experimental Medicine and Biology (E Chantler, J Elder, M Elstein, eds) Plenum Press, New York, 144, 115-133

Allen A, Hutton A, Pearson JP, Sellers LA (1984) Mucus glycoprotein structure, gel formation and gastrointestinal function. In: Mucus and Mucosa (J Nugent, M O'Connor, eds) Pitman, London, Ciba Found Sym 109, 137-151

Bara J, Nardelli J, Dahler N, Decaens (1990) Mucines et cancers. Bull Cancer 77, 390-399

Bensadoun A, Weinstein D (1976) Assay of protein in the presence of interfering materials. Anal Biochem 70, 241-250

Biol MC, Martin A, Louisot PV (1992) Nutritional and developmental regulation of glycosylation processes in digestive organs. Biochem $74,13-24$

Bogomoletz WV, Filipe MI, Potet F (1984) Intérêt de l'histochimie des mucines dans le tube digestif normal et pathologique. Gastroentérol Clin Biol 8, 364-372

Bogomoletz WV, Williams GT, Potet F (1987) Hid-bleu Alcian ("high iron diamine-Alcian blue") et histochimie des mucines en pathologie colique : vingt ans après. Gastroentérol Clin Biol 11, 865-868

Carlstedt I, Sheehan JK (1984) Macromolecular properties and polymeric structure of mucus glycoproteins. In: Mucus and Mucosa. Ciba Foundation Symposium (J Nugent, M O'Connor, eds) Pittman, London, 109, 157-172

Carlstedt I, Sheerham JK, Corfield AP, Gallagher JT (1985) Structure of mucus glycoproteins. A set of problems. Assays in Biochemistry 20, 40-76

Creeth JM (1978) Constituents of mucus and their separation. Br Med Bull 34, 17-24

Doehrn S, Breipohl W, Lierse W, Romaniuk K, Young W (1992) Developmental changes in the distribution of cecal lectin-binding sites of Balb-c mice. Acta Anat 145, 296-301

Ellinger A, Pavelka M (1992) Subdomains of the rough endoplasmic reticulum in colon goblet cells of the rat: Lectin-cytochemical characterisation. $J$ Histochem Cytochem 40, 919930

Fairbanks G, Steck TL, Wallach DFH (1971) Electrophoretic analysis of the major polypeptides of the human erythrocyte membrane. Biochem $10,2606-2617$

Filipe MI (1979) Mucins in the human gastrointestinal epithelium: a review. Invest Cell Pathol 2, 195-216

Filipe MI, Branfoot BM (1974) Abnormal patterns of mucus secretion in apparently normal mucosa of the large intestine with carcinoma. Cancer 34, 282-290

Filipe MI, Fenger C (1979) Histological characteristics of mucins in the small intestine. A comparative study of normal mucosa, begnin epithelial tumors and carcinoma. Histochem $J$ 11, 277-287

Forstner JF, Jabbal I, Forstner GG (1973) Goblet cell mucin of rat small intestine. Chemical and physical characterisation. Can J Biochem 51, 1154-1166

Ganter P, Jollès G (1969-1970) Histochimie normale et pathologique. Vol 1, vol 2, GauthierVillars, Paris

Garner A, Flemström G, Allen A (1984) Gastroduodenal alkaline and mucus secretions. In: Basic Science in Gastroenterology Physiology of the Gut (JM Polak, SR Bloom, NA Wright, AG Butler, eds) Glaxo, Paris, 2nd ed, 207-223

Heremans JF, Vaerman JP, Heremans MTH (1959) Acid mucopolysaccharides of normal urine. Nature 183, 1606

Hilkens J, Ligtenberg MJL, Vos HL, Litvinov SV (1992) Cell membrane-associated mucins and their adhesion-modulating property. Trends Biochem Sci 17, 359-363

Hill RRH (1986) Digestion of mucin polysaccharides in vitro by bacteria isolated from the rabbit cecum. Curr Microbiol 14, 117-120

Hoskins LC, Zamcheck N (1968) Bacterial degradation of gastrointestinal mucins: I. Comparison of mucus constituents in the stools of germ-free and conventional rats. Gastroenterology $54,210-217$

Ishikawa N, Horii Y, Nawa Y (1993) Immunemediated alteration of the terminal sugars of goblet cell mucins in the small intestine of Nippostrongylus brasiliensis-infected rats. Immunology 78, 303-307

Laboisse C, Bogomoletz WV (1989) Les mucines : des glycoprotéines en quête de reconnaissance. Ann Pathol 9, 175-181

Laemmli UK (1970) Cleavage of structural proteins during the assembly of the head of bacteriophage T4. Nature 227, 680-685 
Lambin D, Roch U, Fine JM (1976) A new method for determination of molecular weights of proteins by electrophoresis across a sodium dodecyl sulfate (SDS) polyacrylamide gradient gel. Anal Biochem 74, 567-575

Mantle M, Allen A (1978) A colorimetric assay for glycoproteins based on the periodic acid/Schiff stain. Biochem Soc Trans 6 (1), 607-609

Mantle M, Thakore E, Hardin J, Gall DG (1989) Effect of Yersinia enterocolitica on intestinal mucin secretion. Am J Physiol Gastrointestinal Liver Physiol 19, G319-G327

Maoret JJ, Font J, Augeron C, Codogno P, Banvy C, Aubery M, Laboisse CL (1989) A mucussecreting human colonic cancer cell line; purification and partial characterisation of the secreted mucins. Biochem J 258, 793-799

Meslin JC, Andrieux C, Sakata T, Beaumatin P, Bensaada M, Popot F, Szylit O, Durand M (1993) Effects of galacto-oligosaccharide and bacterial status on mucin distribution in mucosa and on large intestine fermentation in rats. $B r J$ Nutr $69,903-912$

Mian N, Anderson CE, Kent PW (1979) Effect of $O$-sulphated groups in lactose and $\mathrm{N}$-acetyl neuraminyl-lactose on their enzymatic hydrolysis. Biochem J 181, 387-399

Miller RS, Hoskins JC (1981) Mucin degradation in human colon ecosystems. Focal population densities of mucin-degrading bacteria estimated by a "most probable number" method. Gastroenterology 81, 759-765

Neutra MR, Forstner JF (1987) Gastrointestinal Mucus: Synthesis, Secretion and Function. In: Physiology of the Gastrointestinal Tract (LR Johnson, ed) Raven Press, New York, 2nd ed, 34, 975-1009

Pasquier MC, Vatier J (1990) Mucus gastro-intestinal: Une barrière protectrice complexe. Première partie : Structure et propriétés physicochimiques. Gastroentérol Clin Biol 14, 352-358

Perumal AS, Ananthasamy TS, Laksmanan MR, Jungalwala FB, Ramarao PB (1966) Biosynthesis of sulfated mucopolysaccharides in relation to vitamins $A$ and $K . B B A 124,95-$ 100
Porchet N, Dufossé J, Degand P, Aubert JP (1991) Les mucines humaines : pourquoi une telle hétérogénéité peptidique ? MS Med Sci 7, 1024-1030

Rhodes JM (1989) Colonic mucus and mucosal glycoproteins: the key to colitis and cancer? Gut 30, 1660-1666

Roe R, Corfield AP, Williamson RCN (1989) Sialic acid in colonic mucin: an evaluation of modified PAS reactions in single and combination histochemical procedures. Histochem $J 21$, 216-222

Roumagnac I, Laboisse CL (1989) A simple immunofiltration assay for mucins secreted by a human colonic epithelial cell line. J Immunol Methods 122, 265-272

Roussel P, Lamblin G, Lhermitte M, Houdret N, Lafitte JJ, Perini JM, Klein A, Scharfman A (1988) The complexity of mucins. Biochimie $70,1471-1482$

Satchithanandam S, Vargofcak-Apker M, Calvert RJ, Leeds AR, Cassidy MM (1990) Alteration of gastrointestinal mucin by fiber feeding in rats. J Nutr 120, 1179-1184

Snedecor GW, Cochran WG (1967) Statistical Methods. University Press, Ames, 10

Suzaki E, Kataoka K (1992) Lectin cytochemistry in the gastrointestinal tract with special reference to glycosylation in the Golgi apparatus of Brunner's gland cells. J Histochem Cytochem $40,379-385$

Szentkuti L, Staacke S, Busche R (1992) Light microscopic lectin histochemistry on celloidin stabilized cryostat sections of rat colon. Biotech Histochem 67, 360-362

Turck D, Feste AS, Lifschitz CH (1993) Age and diet affect the composition of porcine colonic mucins. Pediatr Res 33, 564-567

Yamori T, Ota DM, Cleary KR, Hoff S, Hager LG, Irimura T (1989) Monoclonal antibody against human colonic sulfomucin: immunochemical detection of its binding sites in colonic mucosa, colorectal primary carcinoma and metastases. Cancer Res 49, 887-894

Zacharius RM, Zell TE, Morrison JH, Woodlock JJ (1969) Glycoprotein staining following electrophoresis on acrylamide gels. Anal Biochem $30,148-152$ 